Article

\title{
Some Inequalities for g-Frames in Hilbert $C^{*}$-Modules
}

\author{
Zhong-Qi Xiang \\ College of Mathematics and Computer Science, Shangrao Normal University, Shangrao 334001, China; \\ 1xsy20110927@163.com; Tel.: +86-793-815-9108
}

Received: 8 November 2018; Accepted: 26 December 2018 ; Published: 27 December 2018

\begin{abstract}
In this paper, we obtain new inequalities for g-frames in Hilbert $C^{*}$-modules by using operator theory methods, which are related to a scalar $\lambda \in \mathbb{R}$ and an adjointable operator with respect to two g-Bessel sequences. It is demonstrated that our results can lead to several known results on this topic when suitable scalars and g-Bessel sequences are chosen.
\end{abstract}

Keywords: Hilbert $C^{*}$-module; g-frame; g-Bessel sequence; adjointable operator

MSC: 46L08; 42C15; 47B48; 46H25

\section{Introduction}

Since their appearance in the literature [1] on nonharmonic Fourier series, frames for Hilbert spaces have been a useful tool and applied to different branches of mathematics and other fields. For details on frames, the reader can refer to the papers [2-11]. The author in [12] extended the concept of frames to bounded linear operators and thus gave us the notion of g-frames, which possess some properties that are quite different from those of frames (see $[13,14]$ ).

In the past decade, much attention has been paid to the extension of frame and $g$-frame theory from Hilbert spaces to Hilbert $C^{*}$-modules, and some significant results have been presented (see [15-23]). It should be pointed out that, due to the essential differences between Hilbert spaces and Hilbert $C^{*}$-modules and the complex structure of the $C^{*}$-algebra involved in a Hilbert $C^{*}$-module, the problems on frames and $g$-frames for Hilbert $C^{*}$-modules are expected to be more complicated than those for Hilbert spaces. Also, increasingly more evidence is indicating that there is a close relationship between the theory of wavelets and frames and Hilbert $C^{*}$-modules in many aspects. This suggests that the discussion of frame and g-frame theory in Hilbert $C^{*}$-modules is interesting and important.

The authors in [24] provided a surprising inequality while further discussing the remarkable identity for Parseval frames derived from their research on effective algorithms to compute the reconstruction of a signal, which was later generalized to the situation of general frames and dual frames [25]. Those inequalities have already been extended to several generalized versions of frames in Hilbert spaces [26-28]. Moreover, the authors in [29-31] showed that g-frames in Hilbert $C^{*}$-modules have their inequalities based on the work in [24,25]; it is worth noting that the inequalities given in [30] are associated with a scalar in $[0,1]$ or $\left[\frac{1}{2}, 1\right]$. In this paper, we establish several new inequalities for g-frames in Hilbert $C^{*}$-modules, where a scalar $\lambda$ in $\mathbb{R}$, the real number set, and an adjointable operator with respect to two g-Bessel sequences are involved. Also, we show that some corresponding results in $[29,31]$ can be considered a special case of our results.

We continue with this section for a review of some notations and definitions.

This paper adopts the following notations: $\mathbb{J}$ and $\mathcal{A}$ are, respectively, a finite or countable index set and a unital $C^{*}$-algebra; $\mathcal{H}, \mathcal{K}$, and $\mathcal{K}_{j}$ 's $\left(j \in \mathbb{J}\right.$ ) are Hilbert $C^{*}$-modules over $\mathcal{A}$ (or simply Hilbert $\mathcal{A}$-modules), setting $\langle f, f\rangle=|f|^{2}$ for any $f \in \mathcal{H}$. The family of all adjointable operators from $\mathcal{H}$ to $\mathcal{K}$ is designated $\operatorname{End}_{\mathcal{A}}^{*}(\mathcal{H}, \mathcal{K})$, which is abbreviated to $\operatorname{End}_{\mathcal{A}}^{*}(\mathcal{H})$ if $\mathcal{K}=\mathcal{H}$. 
A sequence $\Lambda=\left\{\Lambda_{j} \in \operatorname{End}_{\mathcal{A}}^{*}\left(\mathcal{H}, \mathcal{K}_{j}\right)\right\}_{j \in \mathbb{J}}$ denotes a g-frame for $\mathcal{H}$ with respect to $\left\{\mathcal{K}_{j}\right\}_{j \in \mathbb{J}}$ if there are real numbers $0<C \leq D<\infty$ satisfying

$$
C\langle f, f\rangle \leq \sum_{j \in \mathbb{J}}\left\langle\Lambda_{j} f, \Lambda_{j} f\right\rangle \leq D\langle f, f\rangle, \quad \forall f \in \mathcal{H}
$$

If only the second inequality in Equation (1) is required, then $\Lambda$ is said to be a g-Bessel sequence.

For a given g-frame $\Lambda=\left\{\Lambda_{j} \in \operatorname{End}_{\mathcal{A}}^{*}\left(\mathcal{H}, \mathcal{K}_{j}\right)\right\}_{j \in \mathbb{J}}$, there is always a positive, invertible, and self-adjoint operator in $\operatorname{End}_{\mathcal{A}}^{*}(\mathcal{H})$, which we call the g-frame operator of $\Lambda$, defined by

$$
S_{\Lambda}: \mathcal{H} \rightarrow \mathcal{H}, \quad S_{\Lambda} f=\sum_{j \in \mathbb{J}} \Lambda_{j}^{*} \Lambda_{j} f
$$

For any $\mathbb{I} \subset \mathbb{J}$, let $\mathbb{I}^{c}$ be the complement of $\mathbb{I}$. We define a positive and self-adjoint operator in $\operatorname{End}_{\mathcal{A}}^{*}(\mathcal{H})$ related to $\mathbb{I}$ and a g-frame $\Lambda=\left\{\Lambda_{j} \in \operatorname{End}_{\mathcal{A}}^{*}\left(\mathcal{H}, \mathcal{K}_{j}\right)\right\}_{j \in \mathbb{J}}$ in the following form

$$
S_{\mathbb{I}}^{\Lambda}: \mathcal{H} \rightarrow \mathcal{H}, \quad S_{\mathbb{I}}^{\Lambda} f=\sum_{j \in \mathbb{I}} \Lambda_{j}^{*} \Lambda_{j} f .
$$

Recall that a g-Bessel $\Gamma=\left\{\Gamma_{j} \in \operatorname{End}_{\mathcal{A}}^{*}\left(\mathcal{H}, \mathcal{K}_{j}\right)\right\}_{j \in \mathbb{J}}$ is an alternate dual g-frame of $\Lambda$ if, for every $f \in \mathcal{H}$, we have $f=\sum_{j \in \mathbb{J}} \Lambda_{j}^{*} \Gamma_{j} f$.

Let $\Lambda=\left\{\Lambda_{j}\right\}_{j \in \mathbb{J}}$ and $\Gamma=\left\{\Gamma_{j}\right\}_{j \in \mathbb{J}}$ be g-Bessel sequences for $\mathcal{H}$ with respect to $\left\{\mathcal{K}_{j}\right\}_{j \in \mathbb{J}}$. We observe from the Cauchy-Schwarz inequality that the operator

$$
S_{\Gamma \Lambda}: \mathcal{H} \rightarrow \mathcal{H}, \quad S_{\Gamma \Lambda} f=\sum_{j \in \mathbb{I}} \Gamma_{j}^{*} \Lambda_{j} f
$$

is well defined, and a direct calculation shows that $S_{\Gamma \Lambda} \in \operatorname{End}_{\mathcal{A}}^{*}(\mathcal{H})$.

\section{The Main Results}

The following result for operators is used to prove our main results.

Lemma 1. Suppose that $U, V, L \in \operatorname{End}_{\mathcal{A}}^{*}(\mathcal{H})$ and that $U+V=L$. Then, for any $\lambda \in \mathbb{R}$, we have

$$
U^{*} U+\frac{\lambda}{2}\left(V^{*} L+L^{*} V\right)=V^{*} V+\left(1-\frac{\lambda}{2}\right)\left(U^{*} L+L^{*} U\right)+(\lambda-1) L^{*} L \geq\left(\lambda-\frac{\lambda^{2}}{4}\right) L^{*} L .
$$

Proof. On the one hand, we obtain

$$
U^{*} U+\frac{\lambda}{2}\left(V^{*} L+L^{*} V\right)=U^{*} U+\frac{\lambda}{2}\left(\left(L^{*}-U^{*}\right) L+L^{*}(L-U)\right)=U^{*} U-\frac{\lambda}{2}\left(U^{*} L+L^{*} U\right)+\lambda L^{*} L .
$$

On the other hand, we have

$$
\begin{aligned}
V^{*} V & +\left(1-\frac{\lambda}{2}\right)\left(U^{*} L+L^{*} U\right)+(\lambda-1) L^{*} L \\
& =\left(L^{*}-U^{*}\right)(L-U)+\left(U^{*} L+L^{*} U\right)-\frac{\lambda}{2}\left(U^{*} L+L^{*} U\right)+(\lambda-1) L^{*} L \\
& =L^{*} L-\left(U^{*} L+L^{*} U\right)+U^{*} U+\left(U^{*} L+L^{*} U\right)-\frac{\lambda}{2}\left(U^{*} L+L^{*} U\right)+(\lambda-1) L^{*} L \\
& =U^{*} U-\frac{\lambda}{2}\left(U^{*} L+L^{*} U\right)+\lambda L^{*} L=\left(U-\frac{\lambda}{2} L\right)^{*}\left(U-\frac{\lambda}{2} L\right)+\left(\lambda-\frac{\lambda^{2}}{4}\right) L^{*} L \geq\left(\lambda-\frac{\lambda^{2}}{4}\right) L^{*} L .
\end{aligned}
$$

This completes the proof. 
Theorem 1. Let $\Lambda=\left\{\Lambda_{j}\right\}_{j \in \mathbb{J}}$ be a g-frame for $\mathcal{H}$ with respect to $\left\{\mathcal{K}_{j}\right\}_{j \in \mathbb{J}}$. Suppose that $\Gamma=\left\{\Gamma_{j}\right\}_{j \in \mathbb{J}}$ and $\Theta=\left\{\Theta_{j}\right\}_{j \in \mathbb{J}}$ are two g-Bessel sequences for $\mathcal{H}$ with respect to $\left\{\mathcal{K}_{j}\right\}_{j \in \mathbb{J}}$, and that the operator $S_{\Gamma \Lambda}$ is defined in Equation (4). Then, for any $\lambda \in \mathbb{R}$ and any $f \in \mathcal{H}$, we have

$$
\begin{aligned}
\left|\sum_{j \in \mathbb{J}}\left(\Gamma_{j}-\Theta_{j}\right)^{*} \Lambda_{j} f\right|^{2}+\sum_{j \in \mathbb{J}}\left\langle\Lambda_{j} f, \Theta_{j} S_{\Gamma \Lambda} f\right\rangle=\left|\sum_{j \in \mathbb{J}} \Theta_{j}^{*} \Lambda_{j} f\right|^{2}+\sum_{j \in \mathbb{J}}\left\langle\left(\Gamma_{j}-\Theta_{j}\right) S_{\Gamma \Lambda} f, \Lambda_{j} f\right\rangle \\
\geq\left(\lambda-\frac{\lambda^{2}}{4}\right) \sum_{j \in \mathbb{J}}\left\langle\Lambda_{j} f,\left(\Gamma_{j}-\Theta_{j}\right) S_{\Gamma \Lambda} f\right\rangle+\left(1+\frac{\lambda}{2}-\frac{\lambda^{2}}{4}\right) \sum_{j \in \mathbb{J}}\left\langle\Lambda_{j} f, \Theta_{j} S_{\Gamma \Lambda} f\right\rangle \\
\quad-\frac{\lambda}{2} \sum_{j \in \mathbb{J}}\left\langle\Theta_{j} S_{\Gamma \Lambda} f, \Lambda_{j} f\right\rangle .
\end{aligned}
$$

Proof. We let

$$
U f=\sum_{j \in \mathbb{J}}\left(\Gamma_{j}-\Theta_{j}\right)^{*} \Lambda_{j} f \quad \text { and } \quad V f=\sum_{j \in \mathbb{J}} \Theta_{j}^{*} \Lambda_{j} f
$$

for each $f \in \mathcal{H}$. Then, $U, V \in \operatorname{End}_{\mathcal{A}}^{*}(\mathcal{H})$ and, further,

$$
U f+V f=\sum_{j \in \mathbb{J}}\left(\Gamma_{j}-\Theta_{j}\right)^{*} \Lambda_{j} f+\sum_{j \in \mathbb{J}} \Theta_{j}^{*} \Lambda_{j} f=\sum_{j \in \mathbb{J}} \Gamma_{j}^{*} \Lambda_{j} f=S_{\Gamma \Lambda} f .
$$

By Lemma 1, we get

$$
\begin{aligned}
|U f|^{2} & +\frac{\lambda}{2}\left(\left\langle V f, S_{\Gamma \Lambda} f\right\rangle+\left\langle S_{\Gamma \Lambda} f, V f\right\rangle\right) \\
& =|V f|^{2}+\left(1-\frac{\lambda}{2}\right)\left(\left\langle U f, S_{\Gamma \Lambda} f\right\rangle+\left\langle S_{\Gamma \Lambda} f, U f\right\rangle\right)+(\lambda-1)\left|S_{\Gamma \Lambda} f\right|^{2} .
\end{aligned}
$$

Hence,

$$
\begin{aligned}
|U f|^{2}= & |V f|^{2}+\left(1-\frac{\lambda}{2}\right)\left(\left\langle U f, S_{\Gamma \Lambda} f\right\rangle+\left\langle S_{\Gamma \Lambda} f, U f\right\rangle\right)+(\lambda-1)\left|S_{\Gamma \Lambda} f\right|^{2} \\
& -\frac{\lambda}{2}\left(\left\langle V f, S_{\Gamma \Lambda} f\right\rangle+\left\langle S_{\Gamma \Lambda} f, V f\right\rangle\right) \\
= & |V f|^{2}+\left\langle U f, S_{\Gamma \Lambda} f\right\rangle+\left\langle S_{\Gamma \Lambda} f, U f\right\rangle-\frac{\lambda}{2}\left(\left\langle U f, S_{\Gamma \Lambda} f\right\rangle+\left\langle S_{\Gamma \Lambda} f, U f\right\rangle\right) \\
& -\frac{\lambda}{2}\left(\left\langle V f, S_{\Gamma \Lambda} f\right\rangle+\left\langle S_{\Gamma \Lambda} f, V f\right\rangle\right)+(\lambda-1)\left|S_{\Gamma \Lambda} f\right|^{2} \\
= & |V f|^{2}+\left\langle U f, S_{\Gamma \Lambda} f\right\rangle+\left\langle S_{\Gamma \Lambda} f, U f\right\rangle-\frac{\lambda}{2}\left(\left\langle U f, S_{\Gamma \Lambda} f\right\rangle+\left\langle V f, S_{\Gamma \Lambda} f\right\rangle\right) \\
& -\frac{\lambda}{2}\left(\left\langle S_{\Gamma \Lambda} f, U f\right\rangle+\left\langle S_{\Gamma \Lambda} f, V f\right\rangle\right)+(\lambda-1)\left|S_{\Gamma \Lambda} f\right|^{2} \\
= & |V f|^{2}+\left\langle U f, S_{\Gamma \Lambda} f\right\rangle+\left\langle S_{\Gamma \Lambda} f, U f\right\rangle-\lambda\left|S_{\Gamma \Lambda} f\right|^{2}+(\lambda-1)\left|S_{\Gamma \Lambda} f\right|^{2} \\
= & |V f|^{2}+\left\langle U f, S_{\Gamma \Lambda} f\right\rangle+\left\langle S_{\Gamma \Lambda} f, U f\right\rangle-\left\langle U f, S_{\Gamma \Lambda} f\right\rangle-\left\langle V f, S_{\Gamma \Lambda} f\right\rangle .
\end{aligned}
$$

It follows that

$$
|U f|^{2}+\left\langle V f, S_{\Gamma \Lambda} f\right\rangle=|V f|^{2}+\left\langle S_{\Gamma \Lambda} f, U f\right\rangle
$$

from which we arrive at

$$
\left|\sum_{j \in \mathbb{J}}\left(\Gamma_{j}-\Theta_{j}\right)^{*} \Lambda_{j} f\right|^{2}+\sum_{j \in \mathbb{J}}\left\langle\Lambda_{j} f, \Theta_{j} S_{\Gamma \Lambda} f\right\rangle=\left|\sum_{j \in \mathbb{J}} \Theta_{j}^{*} \Lambda_{j} f\right|^{2}+\sum_{j \in \mathbb{J}}\left\langle\left(\Gamma_{j}-\Theta_{j}\right) S_{\Gamma \Lambda} f, \Lambda_{j} f\right\rangle .
$$

We are now in a position to prove the inequality in Equation (5). 
Again by Lemma 1,

$$
\begin{aligned}
|U f|^{2} & \geq\left(\lambda-\frac{\lambda^{2}}{4}\right)\left|S_{\Gamma \Lambda} f\right|^{2}-\frac{\lambda}{2}\left(\left\langle V f, S_{\Gamma \Lambda} f\right\rangle+\left\langle S_{\Gamma \Lambda} f, V f\right\rangle\right) \\
& =\left(\lambda-\frac{\lambda^{2}}{4}\right)\left\langle U f, S_{\Gamma \Lambda} f\right\rangle+\left(\lambda-\frac{\lambda^{2}}{4}\right)\left\langle V f, S_{\Gamma \Lambda} f\right\rangle-\frac{\lambda}{2}\left\langle V f, S_{\Gamma \Lambda} f\right\rangle-\frac{\lambda}{2}\left\langle S_{\Gamma \Lambda} f, V f\right\rangle \\
& =\left(\lambda-\frac{\lambda^{2}}{4}\right)\left\langle U f, S_{\Gamma \Lambda} f\right\rangle+\left(\frac{\lambda}{2}-\frac{\lambda^{2}}{4}\right)\left\langle V f, S_{\Gamma \Lambda} f\right\rangle-\frac{\lambda}{2}\left\langle S_{\Gamma \Lambda} f, V f\right\rangle .
\end{aligned}
$$

Therefore,

$$
\begin{aligned}
& \left|\sum_{j \in \mathbb{J}}\left(\Gamma_{j}-\Theta_{j}\right)^{*} \Lambda_{j} f\right|^{2}+\sum_{j \in \mathbb{J}}\left\langle\Lambda_{j} f, \Theta_{j} S_{\Gamma \Lambda} f\right\rangle=|U f|^{2}+\left\langle V f, S_{\Gamma \Lambda} f\right\rangle \\
& \quad \geq\left(\lambda-\frac{\lambda^{2}}{4}\right)\left\langle U f, S_{\Gamma \Lambda} f\right\rangle+\left(1+\frac{\lambda}{2}-\frac{\lambda^{2}}{4}\right)\left\langle V f, S_{\Gamma \Lambda} f\right\rangle-\frac{\lambda}{2}\left\langle S_{\Gamma \Lambda} f, V f\right\rangle \\
& \quad=\left(\lambda-\frac{\lambda^{2}}{4}\right) \sum_{j \in \mathbb{J}}\left\langle\Lambda_{j} f,\left(\Gamma_{j}-\Theta_{j}\right) S_{\Gamma \Lambda} f\right\rangle+\left(1+\frac{\lambda}{2}-\frac{\lambda^{2}}{4}\right) \sum_{j \in \mathbb{J}}\left\langle\Lambda_{j} f, \Theta_{j} S_{\Gamma \Lambda} f\right\rangle-\frac{\lambda}{2} \sum_{j \in \mathbb{J}}\left\langle\Theta_{j} S_{\Gamma \Lambda} f, \Lambda_{j} f\right\rangle
\end{aligned}
$$

for any $f \in \mathcal{H}$.

Corollary 1. Suppose that $\Lambda=\left\{\Lambda_{j}\right\}_{j \in \mathbb{J}}$ is a g-frame for $\mathcal{H}$ with respect to $\left\{\mathcal{K}_{j}\right\}_{j \in \mathbb{J}}$ with g-frame operator $S_{\Lambda}$ and that $\tilde{\Lambda}_{j}=\Lambda_{j} S_{\Lambda}^{-1}$ for each $j \in \mathbb{J}$. Then, for any $\lambda \in \mathbb{R}$, for all $\mathbb{I} \subset \mathbb{I}$ and all $f \in \mathcal{H}$, we have

$$
\begin{gathered}
\sum_{j \in \mathbb{I}}\left\langle\Lambda_{j} f, \Lambda_{j} f\right\rangle+\sum_{j \in \mathbb{I}}\left\langle\tilde{\Lambda}_{j} S_{\mathbb{I}^{c}}^{\Lambda} f, \tilde{\Lambda}_{j} S_{\mathbb{I}^{c}}^{\Lambda} f\right\rangle=\sum_{j \in \mathbb{I}}\left\langle\Lambda_{j} f, \Lambda_{j} f\right\rangle+\sum_{j \in \mathbb{I}}\left\langle\tilde{\Lambda}_{j} S_{\mathbb{I}}^{\Lambda} f, \tilde{\Lambda}_{j} S_{\mathbb{I}}^{\Lambda} f\right\rangle \\
\geq\left(\lambda-\frac{\lambda^{2}}{4}\right) \sum_{j \in \mathbb{I}^{c}}\left\langle\Lambda_{j} f, \Lambda_{j} f\right\rangle+\left(1-\frac{\lambda^{2}}{4}\right) \sum_{j \in \mathbb{I}}\left\langle\Lambda_{j} f, \Lambda_{j} f\right\rangle .
\end{gathered}
$$

Proof. Taking $\Gamma_{j}=\Lambda_{j} S_{\Lambda}^{-\frac{1}{2}}$ for any $j \in \mathbb{J}$, then it is easy to see that $S_{\Gamma \Lambda}=S_{\Lambda}^{\frac{1}{2}}$. For each $j \in \mathbb{J}$, let

$$
\Theta_{j}= \begin{cases}\Gamma_{j}, & j \in \mathbb{I} \\ 0, & j \in \mathbb{I}^{c}\end{cases}
$$

Now, for each $f \in \mathcal{H}$,

$$
\begin{aligned}
\left|\sum_{j \in \mathbb{J}}\left(\Gamma_{j}-\Theta_{j}\right)^{*} \Lambda_{j} f\right|^{2} & =\left|\sum_{j \in \mathbb{I}^{c}} S_{\Lambda}^{-\frac{1}{2}} \Lambda_{j}^{*} \Lambda_{j} f\right|^{2}=\left|S_{\Lambda}^{-\frac{1}{2}} S_{\mathbb{I}^{c}}^{\Lambda} f\right|^{2}=\left\langle S_{\Lambda}^{-\frac{1}{2}} S_{\mathbb{I}^{c}}^{\Lambda} f, S_{\Lambda}^{-\frac{1}{2}} S_{\mathbb{I}^{c}}^{\Lambda} f\right\rangle \\
& =\left\langle S_{\mathbb{I}^{c}}^{\Lambda} f, S_{\Lambda}^{-1} S_{\mathbb{I}^{c}}^{\Lambda} f\right\rangle=\left\langle S_{\Lambda} S_{\Lambda}^{-1} S_{\mathbb{I}^{c}}^{\Lambda} f, S_{\Lambda}^{-1} S_{\mathbb{I}^{c}}^{\Lambda} f\right\rangle \\
& =\sum_{j \in \mathbb{J}}\left\langle\Lambda_{j} S_{\Lambda}^{-1} S_{\mathbb{I}^{c}}^{\Lambda} f, \Lambda_{j} S_{\Lambda}^{-1} S_{\mathbb{I}^{c}}^{\Lambda} f\right\rangle=\sum_{j \in \mathbb{J}}\left\langle\tilde{\Lambda}_{j} S_{\mathbb{I}^{c}}^{\Lambda} f, \tilde{\Lambda}_{j} S_{\mathbb{I}^{c}}^{\Lambda} f\right\rangle .
\end{aligned}
$$

Since $\left|\sum_{j \in \mathbb{J}} \Theta_{j}^{*} \Lambda_{j} f\right|^{2}=\left|\sum_{j \in \mathbb{I}} \Gamma_{j}^{*} \Lambda_{j} f\right|^{2}=\left|\sum_{j \in \mathbb{I}} S_{\Lambda}^{-\frac{1}{2}} \Lambda_{j}^{*} \Lambda_{j} f\right|^{2}$, a replacement of $\mathbb{I}^{c}$ by $\mathbb{I}$ in the last item of Equation (9) leads to

$$
\left|\sum_{j \in \mathbb{J}} \Theta_{j}^{*} \Lambda_{j} f\right|^{2}=\sum_{j \in \mathbb{J}}\left\langle\tilde{\Lambda}_{j} S_{\mathbb{I}}^{\Lambda} f, \tilde{\Lambda}_{j} S_{\mathbb{I}}^{\Lambda} f\right\rangle .
$$

We also have

$$
\sum_{j \in \mathbb{I}}\left\langle\Lambda_{j} f, \Theta_{j} S_{\Gamma \Lambda} f\right\rangle=\sum_{j \in \mathbb{I}}\left\langle\Lambda_{j} f, \Lambda_{j} f\right\rangle, \quad \sum_{j \in \mathbb{J}}\left\langle\left(\Gamma_{j}-\Theta_{j}\right) S_{\Gamma \Lambda} f, \Lambda_{j} f\right\rangle=\sum_{j \in \mathbb{I}^{c}}\left\langle\Lambda_{j} f, \Lambda_{j} f\right\rangle .
$$


Hence, the conclusion follows from Theorem 1.

Let $\Lambda=\left\{\Lambda_{j}\right\}_{j \in \mathbb{J}}$ be a Parseval g-frame for $\mathcal{H}$ with respect to $\left\{\mathcal{K}_{j}\right\}_{j \in \mathbb{J}} ;$ then, $S_{\Lambda}=\operatorname{Id}_{\mathcal{H}}$. Thus, for any $\mathbb{I} \subset \mathbb{J}$,

$$
\sum_{j \in \mathbb{J}}\left\langle\tilde{\Lambda}_{j} S_{\mathbb{I}^{c}}^{\Lambda} f, \tilde{\Lambda}_{j} S_{\mathbb{I}^{c}}^{\Lambda} f\right\rangle=\sum_{j \in \mathbb{J}}\left\langle\Lambda_{j} S_{\mathbb{I}^{c}}^{\Lambda} f, \Lambda_{j} S_{\mathbb{I}^{c}}^{\Lambda} f\right\rangle=\left|S_{\mathbb{I}^{c}}^{\Lambda} f\right|^{2}=\left|\sum_{j \in \mathbb{I}^{c}} \Lambda_{j}^{*} \Lambda_{j} f\right|^{2}
$$

Similarly,

$$
\sum_{j \in \mathbb{I}}\left\langle\tilde{\Lambda}_{j} S_{\mathbb{I}}^{\Lambda} f, \tilde{\Lambda}_{j} S_{\mathbb{I}}^{\Lambda} f\right\rangle=\left|\sum_{j \in \mathbb{I}} \Lambda_{j}^{*} \Lambda_{j} f\right|^{2} .
$$

This fact, together with Corollary 1, yields

Corollary 2. Suppose that $\Lambda=\left\{\Lambda_{j}\right\}_{j \in \mathbb{J}}$ is a Parseval g-frame for $\mathcal{H}$ with respect to $\left\{\mathcal{K}_{j}\right\}_{j \in \mathbb{J}}$. Then, for any $\lambda \in \mathbb{R}$, for all $\mathbb{I} \subset \mathbb{J}$ and all $f \in \mathcal{H}$, we have

$$
\begin{gathered}
\sum_{j \in \mathbb{I}}\left\langle\Lambda_{j} f, \Lambda_{j} f\right\rangle+\left|\sum_{j \in \mathbb{I}^{c}} \Lambda_{j}^{*} \Lambda_{j} f\right|^{2}=\sum_{j \in \mathbb{I} c}\left\langle\Lambda_{j} f, \Lambda_{j} f\right\rangle+\left|\sum_{j \in \mathbb{I}} \Lambda_{j}^{*} \Lambda_{j} f\right|^{2} \\
\geq\left(\lambda-\frac{\lambda^{2}}{4}\right) \sum_{j \in \mathbb{I}^{c}}\left\langle\Lambda_{j} f, \Lambda_{j} f\right\rangle+\left(1-\frac{\lambda^{2}}{4}\right) \sum_{j \in \mathbb{I}}\left\langle\Lambda_{j} f, \Lambda_{j} f\right\rangle .
\end{gathered}
$$

Corollary 3. Suppose that $\Lambda=\left\{\Lambda_{j}\right\}_{j \in \mathbb{J}}$ is a g-frame for $\mathcal{H}$ with respect to $\left\{\mathcal{K}_{j}\right\}_{j \in \mathbb{J}}$ with an alternate dual g-frame $\Gamma=\left\{\Gamma_{j}\right\}_{j \in \mathbb{J}}$. Then, for any $\lambda \in \mathbb{R}$, for all $\mathbb{I} \subset \mathbb{J}$ and all $f \in \mathcal{H}$, we have

$$
\begin{aligned}
& \left|\sum_{j \in \mathbb{I}} \Gamma_{j}^{*} \Lambda_{j} f\right|^{2}+\sum_{j \in \mathbb{I}^{c}}\left\langle\Lambda_{j} f, \Gamma_{j} f\right\rangle=\left|\sum_{j \in \mathbb{I}^{c}} \Gamma_{j}^{*} \Lambda_{j} f\right|^{2}+\sum_{j \in \mathbb{I}}\left\langle\Gamma_{j} f, \Lambda_{j} f\right\rangle \\
& \quad \geq\left(\lambda-\frac{\lambda^{2}}{4}\right) \sum_{j \in \mathbb{I}}\left\langle\Lambda_{j} f, \Gamma_{j} f\right\rangle+\left(1+\frac{\lambda}{2}-\frac{\lambda^{2}}{4}\right) \sum_{j \in \mathbb{I}^{c}}\left\langle\Lambda_{j} f, \Gamma_{j} f\right\rangle-\frac{\lambda}{2} \sum_{j \in \mathbb{I}^{c}}\left\langle\Gamma_{j} f, \Lambda_{j} f\right\rangle .
\end{aligned}
$$

Proof. We conclude first that $S_{\Gamma \Lambda}=\operatorname{Id}_{\mathcal{H}}$. Now, the result follows immediately from Theorem 1 if, for any $\mathbb{I} \subset \mathbb{J}$, we take $\Theta_{j}= \begin{cases}\Gamma_{j}, & j \in \mathbb{I}^{c}, \\ 0, & j \in \mathbb{I} \text {. }\end{cases}$

Remark 1. Theorems 4.1 and 4.2 in [31] can be obtained if we take $\lambda=1$, respectively, in Corollaries 1 and 2.

Theorem 2. Let $\Lambda=\left\{\Lambda_{j}\right\}_{j \in \mathbb{J}}$ be a g-frame for $\mathcal{H}$ with respect to $\left\{\mathcal{K}_{j}\right\}_{j \in \mathbb{J}}$. Suppose that $\Gamma=\left\{\Gamma_{j}\right\}_{j \in \mathbb{J}}$ and $\Theta=\left\{\Theta_{j}\right\}_{j \in \mathbb{J}}$ are two g-Bessel sequences for $\mathcal{H}$ with respect to $\left\{\mathcal{K}_{j}\right\}_{j \in \mathbb{J}}$ and that the operator $S_{\Gamma \Lambda}$ is defined in Equation (4). Then, for any $\lambda \in \mathbb{R}$ and any $f \in \mathcal{H}$, we have

$$
\begin{gathered}
\left|\sum_{j \in \mathbb{J}}\left(\Gamma_{j}-\Theta_{j}\right)^{*} \Lambda_{j} f\right|^{2}+\left|\sum_{j \in \mathbb{J}} \Theta_{j}^{*} \Lambda_{j} f\right|^{2} \geq\left(\lambda-\frac{\lambda^{2}}{2}\right)\left|\sum_{j \in \mathbb{J}} \Gamma_{j}^{*} \Lambda_{j} f\right|^{2}-(1-\lambda) \sum_{j \in \mathbb{J}}\left\langle\left(\Gamma_{j}-\Theta_{j}\right) S_{\Gamma \Lambda} f, \Lambda_{j} f\right\rangle \\
+(1-\lambda) \sum_{j \in \mathbb{J}}\left\langle\Lambda_{j} f, \Theta_{j} S_{\Gamma \Lambda} f\right\rangle .
\end{gathered}
$$

Moreover, if $U^{*} V$ is positive, where $U$ and $V$ are given in Equation (6), then 


$$
\begin{aligned}
\left|\sum_{j \in \mathbb{J}}\left(\Gamma_{j}-\Theta_{j}\right)^{*} \Lambda_{j} f\right|^{2}+\left|\sum_{j \in \mathbb{J}} \Theta_{j}^{*} \Lambda_{j} f\right|^{2} & \\
& \leq \sum_{j \in \mathbb{J}}\left\langle\left(\Gamma_{j}-\Theta_{j}\right) S_{\Gamma \Lambda} f, \Lambda_{j} f\right\rangle+\sum_{j \in \mathbb{J}}\left\langle\Lambda_{j} f, \Theta_{j} S_{\Gamma \Lambda} f\right\rangle .
\end{aligned}
$$

Proof. Combining Equation (7) with Lemma 1, we obtain

$$
\begin{aligned}
&\left|\sum_{j \in \mathbb{J}}\left(\Gamma_{j}-\Theta_{j}\right)^{*} \Lambda_{j} f\right|^{2}+\left|\sum_{j \in \mathbb{J}} \Theta_{j}^{*} \Lambda_{j} f\right|^{2} \\
&=|U f|^{2}+|V f|^{2}=2|V f|^{2}+\left\langle S_{\Gamma \Lambda} f, U f\right\rangle-\left\langle V f, S_{\Gamma \Lambda} f\right\rangle \\
& \geq\left(2-\frac{\lambda^{2}}{2}\right)\left|S_{\Gamma \Lambda} f\right|^{2}-(2-\lambda)\left(\left\langle S_{\Gamma \Lambda} f, U f\right\rangle+\left\langle U f, S_{\Gamma \Lambda} f\right\rangle\right)+\left\langle S_{\Gamma \Lambda} f, U f\right\rangle-\left\langle V f, S_{\Gamma \Lambda} f\right\rangle \\
&=\left(2-\frac{\lambda^{2}}{2}\right)\left|S_{\Gamma \Lambda} f\right|^{2}-(2-\lambda)\left\langle S_{\Gamma \Lambda} f, U f\right\rangle-(2-\lambda)\left\langle U f, S_{\Gamma \Lambda} f\right\rangle \\
& \quad-(2-\lambda)\left\langle V f, S_{\Gamma \Lambda} f\right\rangle+(1-\lambda)\left\langle V f, S_{\Gamma \Lambda} f\right\rangle+\left\langle S_{\Gamma \Lambda} f, U f\right\rangle \\
&=\left(2-\frac{\lambda^{2}}{2}\right)\left|S_{\Gamma \Lambda} f\right|^{2}-(1-\lambda)\left\langle S_{\Gamma \Lambda} f, U f\right\rangle-(2-\lambda)\left|S_{\Gamma \Lambda} f\right|^{2}+(1-\lambda)\left\langle V f, S_{\Gamma \Lambda} f\right\rangle \\
&=\left(\lambda-\frac{\lambda^{2}}{2}\right)\left|S_{\Gamma \Lambda} f\right|^{2}-(1-\lambda)\left\langle S_{\Gamma \Lambda} f, U f\right\rangle+(1-\lambda)\left\langle V f, S_{\Gamma \Lambda} f\right\rangle \\
&=\left(\lambda-\frac{\lambda^{2}}{2}\right)\left|\sum_{j \in \mathbb{J}} \Gamma_{j}^{*} \Lambda_{j} f\right|^{2}-(1-\lambda) \sum_{j \in \mathbb{J}}\left\langle\left(\Gamma_{j}-\Theta_{j}\right) S_{\Gamma \Lambda} f, \Lambda_{j} f\right\rangle+(1-\lambda) \sum_{j \in \mathbb{J}}\left\langle\Lambda_{j} f, \Theta_{j} S_{\Gamma \Lambda} f\right\rangle
\end{aligned}
$$

for any $f \in \mathcal{H}$. We next prove Equation (12). Since $U^{*} V$ is positive, we see from Equation (7) that

$$
|U f|^{2}=|V f|^{2}+\left\langle S_{\Gamma \Lambda} f, U f\right\rangle-\left\langle V f, S_{\Gamma \Lambda} f\right\rangle=\left\langle S_{\Gamma \Lambda} f, U f\right\rangle-\langle V f, U f\rangle \leq\left\langle S_{\Gamma \Lambda} f, U f\right\rangle
$$

for each $f \in \mathcal{H}$. A similar discussion gives $|V f|^{2} \leq\left\langle V f, S_{\Gamma \Lambda} f\right\rangle$. Thus,

$$
\begin{aligned}
\left|\sum_{j \in \mathbb{J}}\left(\Gamma_{j}-\Theta_{j}\right)^{*} \Lambda_{j} f\right|^{2}+\left|\sum_{j \in \mathbb{J}} \Theta_{j}^{*} \Lambda_{j} f\right|^{2} & =|U f|^{2}+|V f|^{2} \leq\left\langle S_{\Gamma \Lambda} f, U f\right\rangle+\left\langle V f, S_{\Gamma \Lambda} f\right\rangle \\
& =\sum_{j \in \mathbb{J}}\left\langle\left(\Gamma_{j}-\Theta_{j}\right) S_{\Gamma \Lambda} f, \Lambda_{j} f\right\rangle+\sum_{j \in \mathbb{J}}\left\langle\Lambda_{j} f, \Theta_{j} S_{\Gamma \Lambda} f\right\rangle .
\end{aligned}
$$

Corollary 4. Let $\Lambda=\left\{\Lambda_{j}\right\}_{j \in \mathbb{J}}$ be a g-frame for $\mathcal{H}$ with respect to $\left\{\mathcal{K}_{j}\right\}_{j \in \mathbb{J}}$ with g-frame operator $S_{\Lambda}$, and $\tilde{\Lambda}_{j}=\Lambda_{j} S_{\Lambda}^{-1}$ for each $j \in \mathbb{J}$. Then, for any $\lambda \in \mathbb{R}$, for all $\mathbb{I} \subset \mathbb{J}$ and all $f \in \mathcal{H}$, we have

$$
\begin{gathered}
\left(\lambda-\frac{\lambda^{2}}{2}\right) \sum_{j \in \mathbb{J}}\left\langle\Lambda_{j} f, \Lambda_{j} f\right\rangle-(1-\lambda) \sum_{j \in \mathbb{C}}\left\langle\Lambda_{j} f, \Lambda_{j} f\right\rangle+(1-\lambda) \sum_{j \in \mathbb{I}}\left\langle\Lambda_{j} f, \Lambda_{j} f\right\rangle \\
\quad \leq \sum_{j \in \mathbb{J}}\left\langle\tilde{\Lambda}_{j} S_{\mathbb{I}}^{\Lambda} f, \tilde{\Lambda}_{j} S_{\mathbb{I}}^{\Lambda} f\right\rangle+\sum_{j \in \mathbb{J}}\left\langle\tilde{\Lambda}_{j} S_{\mathbb{I}}^{\Lambda} f, \tilde{\Lambda}_{j} S_{\mathbb{I} c}^{\Lambda} f\right\rangle \leq \sum_{j \in \mathbb{J}}\left\langle\Lambda_{j} f, \Lambda_{j} f\right\rangle .
\end{gathered}
$$

Proof. For every $j \in \mathbb{J}$, taking $\Gamma_{j}=\Lambda_{j} S_{\Lambda}^{-\frac{1}{2}}$ and $\Theta_{j}=\left\{\begin{array}{ll}\Gamma_{j}, & j \in \mathbb{I}, \\ 0, & j \in \mathbb{I}^{c},\end{array}\right.$ then the operators $U$ and $V$ defined in Equation (6) can be expressed as $U=S_{\Lambda}^{-\frac{1}{2}} S_{\mathbb{I} c}^{\Lambda}$ and $V=S_{\Lambda}^{-\frac{1}{2}} S_{\mathbb{I}}^{\Lambda}$, respectively. Hence, $U^{*} V=S_{\mathbb{I}}^{\Lambda} S_{\Lambda}^{-1} S_{\mathbb{I}}^{\Lambda}$. Since $S_{\Lambda}^{-\frac{1}{2}} S_{\mathbb{I}}^{\Lambda} S_{\Lambda}^{-\frac{1}{2}}$ and $S_{\Lambda}^{-\frac{1}{2}} S_{\mathbb{I}}^{\Lambda} S_{\Lambda}^{-\frac{1}{2}}$ are positive and commutative, it follows that 


$$
0 \leq S_{\Lambda}^{-\frac{1}{2}} S_{\mathbb{I}}^{\Lambda} S_{\Lambda}^{-\frac{1}{2}} S_{\Lambda}^{-\frac{1}{2}} S_{\mathbb{I}}^{\Lambda} S_{\Lambda}^{-\frac{1}{2}}=S_{\Lambda}^{-\frac{1}{2}} S_{\mathbb{I}}^{\Lambda} S_{\Lambda}^{-1} S_{\mathbb{I}}^{\Lambda} S_{\Lambda}^{-\frac{1}{2}},
$$

and, consequently, $S_{\mathbb{I}}^{\Lambda} S_{\Lambda}^{-1} S_{\mathbb{I}}^{\Lambda} \geq 0$. Note also that

$$
\left|\sum_{j \in \mathbb{J}} \Gamma_{j}^{*} \Lambda_{j} f\right|^{2}=\left|S_{\Lambda}^{-\frac{1}{2}} \sum_{j \in \mathbb{J}} \Lambda_{j}^{*} \Lambda_{j} f\right|^{2}=\left|S_{\Lambda}^{\frac{1}{2}} f\right|^{2}=\left\langle S_{\Lambda} f, f\right\rangle=\sum_{j \in \mathbb{J}}\left\langle\Lambda_{j} f, \Lambda_{j} f\right\rangle .
$$

Now, the result follows by combining Theorem 2 and Equations (9)-(11).

Theorem 3. Let $\Lambda=\left\{\Lambda_{j}\right\}_{j \in \mathbb{J}}$ be a g-frame for $\mathcal{H}$ with respect to $\left\{\mathcal{K}_{j}\right\}_{j \in \mathbb{J}}$ with g-frame operator $S_{\Lambda}$. Suppose that $\Gamma=\left\{\Gamma_{j}\right\}_{j \in \mathbb{J}}$ and $\Theta=\left\{\Theta_{j}\right\}_{j \in \mathbb{J}}$ are two g-Bessel sequences for $\mathcal{H}$ with respect to $\left\{\mathcal{K}_{j}\right\}_{j \in \mathbb{J}}$ and that the operator $S_{\Gamma \Lambda}$ is defined in Equation (4). Then, for any $\lambda \in \mathbb{R}$ and any $f \in \mathcal{H}$, we have

$$
\begin{aligned}
\sum_{j \in \mathbb{J}}\left\langle\Lambda_{j} f, \Theta_{j} S_{\Lambda}^{\frac{1}{2}} f\right\rangle-\left|\sum_{j \in \mathbb{J}} \Theta_{j}^{*} \Lambda_{j} f\right|^{2} \leq \sum_{j \in \mathbb{J}}\left\langle\Lambda_{j} f, \Theta_{j}\left(S_{\Lambda}^{\frac{1}{2}}-S_{\Gamma \Lambda}\right) f\right\rangle-\frac{\lambda}{2} \sum_{j \in \mathbb{J}}\left\langle\Lambda_{j} f,\left(\Gamma_{j}-\Theta_{j}\right) S_{\Gamma \Lambda} f\right\rangle \\
+\left(1-\frac{\lambda}{2}\right) \sum_{j \in \mathbb{J}}\left\langle\left(\Gamma_{j}-\Theta_{j}\right) S_{\Gamma \Lambda} f, \Lambda_{j} f\right\rangle+\frac{\lambda^{2}}{4}\left|\sum_{j \in \mathbb{J}} \Gamma_{j}^{*} \Lambda_{j} f\right|^{2} .
\end{aligned}
$$

Moreover, if $U^{*} V$ is positive, where $U$ and $V$ are given in Equation (6), then

$$
\sum_{j \in \mathbb{J}}\left\langle\Lambda_{j} f, \Theta_{j} S_{\Lambda}^{\frac{1}{2}} f\right\rangle-\left|\sum_{j \in \mathbb{J}} \Theta_{j}^{*} \Lambda_{j} f\right|^{2} \geq \sum_{j \in \mathbb{J}}\left\langle\Lambda_{j} f, \Theta_{j}\left(S_{\Lambda}^{\frac{1}{2}}-S_{\Gamma \Lambda}\right) f\right\rangle .
$$

Proof. Combining Equations (7) and (8) leads to

$$
\begin{aligned}
\sum_{j \in \mathbb{J}}\left\langle\Lambda_{j} f, \Theta_{j} S_{\Lambda}^{\frac{1}{2}} f\right\rangle-\left|\sum_{j \in \mathbb{J}} \Theta_{j}^{*} \Lambda_{j} f\right|^{2}=\left\langle S_{\Lambda}^{\frac{1}{2}} V f, f\right\rangle-|V f|^{2} \\
\leq\left\langle S_{\Lambda}^{\frac{1}{2}} V f, f\right\rangle-\left(\lambda-\frac{\lambda^{2}}{4}\right)\left\langle U f, S_{\Gamma \Lambda} f\right\rangle-\left(\frac{\lambda}{2}-\frac{\lambda^{2}}{4}\right)\left\langle V f, S_{\Gamma \Lambda} f\right\rangle \\
\quad+\frac{\lambda}{2}\left\langle S_{\Gamma \Lambda} f, V f\right\rangle-\left\langle V f, S_{\Gamma \Lambda} f\right\rangle+\left\langle S_{\Gamma \Lambda} f, U f\right\rangle \\
=\left\langle V f,\left(S_{\Lambda}^{\frac{1}{2}}-S_{\Gamma \Lambda}\right) f\right\rangle-\left(\frac{\lambda}{2}-\frac{\lambda^{2}}{4}\right)\left(\left\langle U f, S_{\Gamma \Lambda} f\right\rangle+\left\langle V f, S_{\Gamma \Lambda} f\right\rangle\right)-\frac{\lambda}{2}\left\langle U f, S_{\Gamma \Lambda} f\right\rangle \\
\quad+\frac{\lambda}{2}\left(\left\langle S_{\Gamma \Lambda} f, V f\right\rangle+\left\langle S_{\Gamma \Lambda} f, U f\right\rangle\right)+\left(1-\frac{\lambda}{2}\right)\left\langle S_{\Gamma \Lambda} f, U f\right\rangle \\
=\left\langle V f,\left(S_{\Lambda}^{\frac{1}{2}}-S_{\Gamma \Lambda}\right) f\right\rangle-\left(\frac{\lambda}{2}-\frac{\lambda^{2}}{4}\right)\left|S_{\Gamma \Lambda} f\right|^{2} \\
\quad-\frac{\lambda}{2}\left\langle U f, S_{\Gamma \Lambda} f\right\rangle+\frac{\lambda}{2}\left|S_{\Gamma \Lambda} f\right|^{2}+\left(1-\frac{\lambda}{2}\right)\left\langle S_{\Gamma \Lambda} f, U f\right\rangle \\
=\left\langle V f,\left(S_{\Lambda}^{\frac{1}{2}}-S_{\Gamma \Lambda}\right) f\right\rangle+\frac{\lambda^{2}}{4}\left|S_{\Gamma \Lambda} f\right|^{2}-\frac{\lambda}{2}\left\langle U f, S_{\Gamma \Lambda} f\right\rangle+\left(1-\frac{\lambda}{2}\right)\left\langle S_{\Gamma \Lambda} f, U f\right\rangle \\
=\sum_{j \in \mathbb{J}}\left\langle\Lambda_{j} f, \Theta_{j}\left(S_{\Lambda}^{\frac{1}{2}}-S_{\Gamma \Lambda}\right) f\right\rangle-\frac{\lambda}{2} \sum_{j \in \mathbb{J}}\left\langle\Lambda_{j} f,\left(\Gamma_{j}-\Theta_{j}\right) S_{\Gamma \Lambda} f\right\rangle \\
\quad+\left(1-\frac{\lambda}{2}\right) \sum_{j \in \mathbb{J}}\left\langle\left(\Gamma_{j}-\Theta_{j}\right) S_{\Gamma \Lambda} f, \Lambda_{j} f\right\rangle+\frac{\lambda^{2}}{4}\left|\sum_{j \in \mathbb{J}} \Gamma_{j}^{*} \Lambda_{j} f\right|^{2}, \quad \forall f \in \mathcal{H} .
\end{aligned}
$$


Suppose that $U^{*} V$ is positive; then, $|V f|^{2} \leq\left\langle V f, S_{\Gamma \Lambda} f\right\rangle$. Now, the "Moreover" part follows from the following inequality:

$$
\begin{aligned}
\sum_{j \in \mathbb{J}}\left\langle\Lambda_{j} f, \Theta_{j} S_{\Lambda}^{\frac{1}{2}} f\right\rangle-\left|\sum_{j \in \mathbb{J}} \Theta_{j}^{*} \Lambda_{j} f\right|^{2} & =\left\langle S_{\Lambda}^{\frac{1}{2}} V f, f\right\rangle-|V f|^{2} \geq\left\langle S_{\Lambda}^{\frac{1}{2}} V f, f\right\rangle-\left\langle V f, S_{\Gamma \Lambda} f\right\rangle \\
& =\left\langle V f,\left(S_{\Lambda}^{\frac{1}{2}}-S_{\Gamma \Lambda}\right) f\right\rangle=\sum_{j \in \mathbb{J}}\left\langle\Lambda_{j} f, \Theta_{j}\left(S_{\Lambda}^{\frac{1}{2}}-S_{\Gamma \Lambda}\right) f\right\rangle .
\end{aligned}
$$

Corollary 5. Let $\Lambda=\left\{\Lambda_{j}\right\}_{j \in \mathbb{J}}$ be a g-frame for $\mathcal{H}$ with respect to $\left\{\mathcal{K}_{j}\right\}_{j \in \mathbb{J}}$ with g-frame operator $S_{\Lambda}$. Then, for any $\lambda \in \mathbb{R}$, for all $\mathbb{I} \subset \mathbb{J}$ and all $f \in \mathcal{H}$, we have

$$
\begin{aligned}
0 & \leq \sum_{j \in \mathbb{I}}\left\langle\Lambda_{j} f, \Lambda_{j} f\right\rangle-\sum_{j \in \mathbb{I}}\left\langle\tilde{\Lambda}_{j} S_{\mathbb{I}}^{\Lambda} f, \tilde{\Lambda}_{j} S_{\mathbb{I}}^{\Lambda} f\right\rangle \\
& \leq(1-\lambda) \sum_{j \in \mathbb{I}^{c}}\left\langle\Lambda_{j} f, \Lambda_{j} f\right\rangle+\frac{\lambda^{2}}{4} \sum_{j \in \mathbb{J}}\left\langle\Lambda_{j} f, \Lambda_{j} f\right\rangle .
\end{aligned}
$$

Proof. For each $j \in \mathbb{J}$, let $\Gamma_{j}$ and $\Theta_{j}$ be the same as in the proof of Corollary 4 . By Theorem 3, we have

$$
\begin{aligned}
\sum_{j \in \mathbb{I}}\left\langle\Lambda_{j} f, \Lambda_{j} f\right\rangle-\sum_{j \in \mathbb{J}}\left\langle\tilde{\Lambda}_{j} S_{\mathbb{I}}^{\Lambda} f, \tilde{\Lambda}_{j} S_{\mathbb{I}}^{\Lambda} f\right\rangle=\sum_{j \in \mathbb{I}}\left\langle\Lambda_{j} f, \Theta_{j} S_{\Lambda}^{\frac{1}{2}} f\right\rangle-\left|\sum_{j \in \mathbb{J}} \Theta_{j}^{*} \Lambda_{j} f\right|^{2} \\
\leq-\frac{\lambda}{2} \sum_{j \in \mathbb{I}^{c}}\left\langle\Lambda_{j} f, \Lambda_{j} f\right\rangle+\left(1-\frac{\lambda}{2}\right) \sum_{j \in \mathbb{I}^{c}}\left\langle\Lambda_{j} f, \Lambda_{j} f\right\rangle+\frac{\lambda^{2}}{4} \sum_{j \in \mathbb{J}}\left\langle\Lambda_{j} f, \Lambda_{j} f\right\rangle \\
=(1-\lambda) \sum_{j \in \mathbb{I}^{c}}\left\langle\Lambda_{j} f, \Lambda_{j} f\right\rangle+\frac{\lambda^{2}}{4} \sum_{j \in \mathbb{J}}\left\langle\Lambda_{j} f, \Lambda_{j} f\right\rangle .
\end{aligned}
$$

By Theorem 3 again,

$$
\begin{aligned}
\sum_{j \in \mathbb{I}}\left\langle\Lambda_{j} f, \Lambda_{j} f\right\rangle-\sum_{j \in \mathbb{J}}\left\langle\tilde{\Lambda}_{j} S_{\mathbb{I}}^{\Lambda} f, \tilde{\Lambda}_{j} S_{\mathbb{I}}^{\Lambda} f\right\rangle & =\sum_{j \in \mathbb{J}}\left\langle\Lambda_{j} f, \Theta_{j} S_{\Lambda}^{\frac{1}{2}} f\right\rangle-\left|\sum_{j \in \mathbb{J}} \Theta_{j}^{*} \Lambda_{j} f\right|^{2} \\
& \geq \sum_{j \in \mathbb{J}}\left\langle\Lambda_{j} f, \Theta_{j}\left(S_{\Lambda}^{\frac{1}{2}}-S_{\Gamma \Lambda}\right) f\right\rangle=0,
\end{aligned}
$$

and the proof is finished.

Remark 2. Taking $\lambda=1$ in Corollaries 4 and 5, we can obtain Theorem 2.4 in [29].

Funding: This research was funded by the National Natural Science Foundation of China under grant numbers 11761057 and 11561057.

Conflicts of Interest: The author declares no conflict of interest.

\section{References}

1. Duffin, R.J.; Schaeffer, A.C. A class of nonharmonic Fourier series. Trans. Am. Math. Soc. 1952, 72, $341-366$. [CrossRef]

2. Bemrose, T.; Casazza, P.G.; Gröchenig, K.; Lammers, M.C.; Lynch, R.G. Weaving frames. Oper. Matrices 2016, 10, 1093-1116. [CrossRef]

3. Benedetto, J.; Powell, A.; Yilmaz, O. Sigma-Delta $(\Sigma \Delta)$ quantization and finite frames. IEEE Trans. Inf. Theory 2006, 52, 1990-2005. [CrossRef] 
4. Casazza, P.G. The art of frame theory. Taiwan J. Math. 2000, 4, 129-201. [CrossRef]

5. Christensen, O. An Introduction to Frames and Riesz Bases; Birkhäuser: Boston, MA, USA, 2000.

6. Christensen, O.; Hasannasab, M. Operator representations of frames: Boundedness, duality, and stability. Integral Equ. Oper. Theory 2017, 88, 483-499. [CrossRef]

7. Christensen, O.; Hasannasab, M.; Rashidi, E. Dynamical sampling and frame representations with bounded operators. J. Math. Anal. Appl. 2018, 463, 634-644. [CrossRef]

8. Daubechies, I.; Grossmann, A.; Meyer, Y. Painless nonorthogonal expansions. J. Math. Phys. 1986, 27, 1271-1283. [CrossRef]

9. Han, D.; Sun, W. Reconstruction of signals from frame coefficients with erasures at unknown locations. IEEE Trans. Inf. Theory 2014, 60, 4013-4025. [CrossRef]

10. Strohmer, T.; Heath, R. Grassmannian frames with applications to coding and communication. Appl. Comput. Harmon. Anal. 2003, 14, 257-275. [CrossRef]

11. Sun, W. Asymptotic properties of Gabor frame operators as sampling density tends to infinity. J. Funct. Anal. 2010, 258, 913-932. [CrossRef]

12. Sun, W. G-frames and g-Riesz bases. J. Math. Anal. Appl. 2006, 322, 437-452. [CrossRef]

13. Sun, W. Stability of g-frames. J. Math. Anal. Appl. 2007, 326, 858-868. [CrossRef]

14. Li, J.Z.; Zhu, Y.C. Exact g-frames in Hilbert spaces. J. Math. Anal. Appl. 2011, 374, 201-209. [CrossRef]

15. Frank, M.; Larson, D.R. Frames in Hilbert $C^{*}$-modules and $C^{*}$-algebras. J. Oper. Theory 2002, 48, $273-314$.

16. Arambašić, L. On frames for countably generated Hilbert $C^{*}$-modules. Proc. Am. Math. Soc. 2007, 135, 469-478. [CrossRef]

17. Han, D.; Jing, W.; Larson, D.R.; Li, P.T.; Mohapatra, R.N. Dilation of dual frame pairs in Hilbert $C^{*}$-modules. Results Math. 2013, 63, 241-250. [CrossRef]

18. Arambašić, L.; Bakić, D. Frames and outer frames for Hilbert $C^{*}$-modules. Linear Multilinear Algebra 2017, 65, 381-431. [CrossRef]

19. Khosravi, A.; Khosravi, B. Fusion frames and g-frames in Hilbert $C^{*}$-modules. Int. J. Wavel. Multiresolut. Inf. Process. 2008, 6, 433-446. [CrossRef]

20. Khosravi, A.; Mirzaee Azandaryani, M. Bessel multipliers in Hilbert $C^{*}$-modoles. Banach J. Math. Anal. 2015, 9, 153-163. [CrossRef]

21. Han, D.; Jing, W.; Larson, D.R.; Mohapatra, R.N. Riesz bases and their dual modular frames in Hilbert $C^{*}$-modules. J. Math. Anal. Appl. 2008, 343, 246-256. [CrossRef]

22. Alijani, A.; Dehghan, M.A. G-frames and their duals for Hilbert $C^{*}$-modules. Bull. Iran. Math. Soc. 2012, 38, 567-580.

23. Alijani, A. Generalized frames with $C^{*}$-valued bounds and their operator duals. Filomat 2015, 29, 1469-1479. [CrossRef]

24. Balan, R.; Casazza, P.G.; Edidin, D.; Kutyniok, G. A new identity for Parseval frames. Proc. Am. Math. Soc. 2007, 135, 1007-1015. [CrossRef]

25. Găvruţa, P. On some identities and inequalities for frames in Hilbert spaces. J. Math. Anal. Appl. 2006, 321, 469-478. [CrossRef]

26. Li, D.W.; Leng, J.S. On some new inequalities for fusion frames in Hilbert spaces. Math. Inequal. Appl. 2017, 20, 889-900. [CrossRef]

27. Li, D.W.; Leng, J.S. On some new inequalities for continuous fusion frames in Hilbert spaces. Mediterr. J. Math. 2018, 15, 173. [CrossRef]

28. Poria, A. Some identities and inequalities for Hilbert-Schmidt frames. Mediterr. J. Math. 2017, 14, 59. [CrossRef]

29. Xiang, Z.Q. New inequalities for g-frames in Hilbert $C^{*}$-modules. J. Math. Inequal. 2016, 10, 889-897. [CrossRef] 
30. Xiang, Z.Q. New double inequalities for g-frames in Hilbert $C^{*}$-modules. SpringerPlus 2016, 5, 1025. [CrossRef]

31. Xiao, X.C.; Zeng, X.M. Some properties of g-frames in Hilbert C*-modules. J. Math. Anal. Appl. 2010, 363, 399-408. [CrossRef] 